\title{
DETERMINAÇÃO DO VALOR DE PREVENÇÃO PARA CROMO EM SOLOS DO ESTADO DE MINAS GERAIS
}

É. A. MARQUES
Universidade Federal de Lavras
ericagroeng@yahoo.com.br
Artigo submetido em janeiro/2016 e aceito em julho/2016
DOI: $10.15628 /$ holos.2016.4011

\section{RESUMO}

Este trabalho visa à investigação dos valores de prevenção para $\mathrm{Cr}$ em duas classes de solos. Foram selecionadas duas classes de solos do Estado de moderado textura argilosa (CXbd) proveniente do campus da Universidade Federal de Lavras - Lavras-MG. Os solos foram classificados como Latossolo Vermelho Amarelo distrófico A moderado textura média fase floresta tropical subperenifólia (LVAd) proveniente do Município de Itumirim-MG e Cambissolo Háplico também distrófico típico A As espécies de plantas testadas foram Zea mays e Phaseolus aureus. Os tratamentos consistiram de oito doses de $\operatorname{Cr}(0,5,10,20$, 45, 90, 200 e $400 \mathrm{mg} \mathrm{kg}^{-1}$ de solo seco) aplicados em solução sob a forma de dicromato de K. Os efeitos dos tratamentos sobre as espécies vegetais foram avaliados por meio da medição da altura de parte aérea, comprimento de raiz, massa seca da parte aérea, massa seca de raiz. Os dados foram submetidos a modelos não lineares por meio do programa STATISTIC 7.0. O valor de VP estimado encontrado neste trabalho foi de $292 \mathrm{mg} \mathrm{kg}$ ${ }^{1}$ para CXbd na cultura do milho, enquanto que o valor atual preconizado pela legislação é de $75 \mathrm{mg} \mathrm{kg}^{-1}$. Este valor indica a necessidade de revisão da legislação atual e deve ser usado como ponto de partida para estudos futuros. Podendo-se afirmar que quanto maior as doses de $\mathrm{Cr}$ adicionadas nos solos maiores os teores de $\mathrm{Cr}$ presentes na planta. Apesar das culturas absorverem $\mathrm{Cr}$ em suas estruturas, grande parte deste fica ainda retido solo.

PALAVRAS-CHAVE: Elementos traços, Dicromato de potássio, Valor de prevenção.

\section{DETERMINING THE VALUE OF PREVENTION FOR CHROMIUM IN THE STATE OF MINAS GERAIS SOILS}

\begin{abstract}
This paper aims to investigate the values of prevention for $\mathrm{Cr}$ in two classes of soils. They selected two state soil classes of moderate clayey (CXbd) from the campus of the Federal University of Lavras. The soils were classified as Oxisol medium texture dystrophic The moderate rain forest stage subperenifólia (LVAd) from the Municipality of Itumirim-MG and Cambisol also Hapludox The species of plants tested were Zea mays and Phaseolus aureus. The treatments consisted of eight doses of $\operatorname{Cr}(0,5,10$, $20,45,90,200$ and $400 \mathrm{mg} \mathrm{kg}^{-1}$ of dry soil) applied in solution in the form of dichromate $K$. The effects of treatments on the plants were evaluated by means of
\end{abstract}

shoot height, root length, shoot dry weight, root dry weight. Data were subjected to non-linear models through the STATISTIC 7.0 software. The value of VP estimated in this study was $292 \mathrm{mg} \mathrm{kg}^{-1}$ for CXbd in maize, while the current value recommended by law is $75 \mathrm{mg} \mathrm{kg}$ ${ }^{1}$. This value indicates the need to review the current legislation and should be used as a starting point for future studies. It can be said that the higher the dose of $\mathrm{Cr}$ added in the largest land the $\mathrm{Cr}$ content present in the plant. Although the crops absorb $\mathrm{Cr}$ in their structures, much of this is still retained soil.

KEYWORDS: Trace elements, Potassium dichromate, Value prevention. 


\section{INTRODUÇÃO}

O desenvolvimento de pesquisa na área agrícola e ambiental tem reconhecido o importante papel do solo no meio ambiente. Estabeleceu-se a percepção deste como componente ativo, atuando não somente como um filtro e/ou dreno para contaminantes, mas também como um tampão natural controlando o transporte de elementos químicos e diversas outras substâncias para a atmosfera, hidrosfera e biosfera (KABATA-BENDIAS, 2011).

Um importante instrumento para prevenção e controle da contaminação e gerenciamento de áreas contaminadas são os valores orientadores que servem como norteadores para a qualidade do solo e de águas subterrâneas. Estes são divididos em três categorias: Valor de Referência de Qualidade ou background (VRQ), Valor de Prevenção (VP) e Valor de Intervenção (VI), regulamentados pela Deliberação Normativa Conjunta COPAM/CERH-MG № 02, de 08/09/2010 e pela Deliberação Normativa COPAM-MG no 166, de 29/06/2011.

Dentre os ETs de relevância ambiental, o $\mathrm{Cr}$ se destaca por ser muito utilizado pelas indústrias metalúrgicas, em fertilizantes, curtimento de couro e materiais de limpeza. Se não descartado de forma correta, pode contaminar rios, lagos e solos. Comumente, o $\mathrm{Cr}$ encontra-se na natureza nos estados de oxidação II e III. O Cr(VI) ocorre geralmente associado ao oxigênio na forma de oxiânions como o cromato (CrO42-) ou dicromato ( $\mathrm{Cr} 2 \mathrm{O} 72-$ ). O Cr (III) é encontrado em ambientes aquáticos na forma de $\mathrm{Cr} 3+$ e ligado à matéria orgânica do solo. Com relação ao seu comportamento no solo, o $\mathrm{Cr}(\mathrm{VI})$ é um oxiânion que apresenta maior solubilidade e mobilidade no solo e nos sistemas aquáticos, sendo muitas vezes mais tóxico que o $\mathrm{Cr}$ (III) (DETTMER et al., 2010).

Para se estabelecer os valores orientadores leva-se em conta os elementos traços (ETs) que formam um grupo de substâncias químicas com particularidades relevantes e de ocorrência natural em rochas e no ambiente, podendo haver um incremento pelas atividades antrópicas (BIONDI, 2010). Apesar da essencialidade dos ETs ser comprovada para algumas plantas e animais, quando encontrados acima de limites permissíveis estes podem causar diversos desequilíbrios ecológicos (PELOZATO, 2008).

O Cr pode ser considerado relativamente abundante, com uma concentração média de 100 $\mathrm{mg} \mathrm{kg}{ }^{-1}$ e está presente principalmente em rochas ultramáficas e máficas. O VRQ de $\mathrm{Cr}$ no Estado de Minas Gerais é de $75 \mathrm{mg} \mathrm{kg}^{-1}$ (peso seco) (Minas Gerais, 2010). Sendo a absorção de $\mathrm{Cr}$ pelas plantas associadas às características da cultura e do meio onde a planta se desenvolve (SHANKER et al., 2005).

A absorção de $\mathrm{Cr}$ pelas plantas está associada às características da cultura e do meio onde as planta se desenvolvem. Nesse sentido, o estado de oxidação do elemento no solo exerce importante papel. Na forma hexavalente, o $\mathrm{Cr}$ apresenta-se como cromato, sendo solúvel, de penetração fácil através da membrana celular e apresentando uma ação tóxica aguda por ser um forte agente oxidante (MARTINES et al., 2008). O $\mathrm{Cr}$ é absorvido e acumulado pelas raízes juntamente com o Fe (III), sendo pouco translocado para a parte aérea.

A concentração de $\mathrm{Cr}$ na parte aérea das plantas é cem vezes menor que nas raízes, independentemente da espécie. Os teores em plantas de solos não contaminados são comumente 
menores que $1 \mathrm{mg} \mathrm{kg}^{-1}$, raramente excedendo $5 \mathrm{mg} \mathrm{kg}^{-1}$, e tipicamente na ordem de 0,02-0,2 $\mathrm{mg} \mathrm{kg}^{-1}$. O Cr ( VI) é tóxico para a maioria das culturas em níveis que variam de 5 a $100 \mathrm{mg} \mathrm{kg}^{-1} \mathrm{em}$ solos. Neste contexto a proteção do solo deve ser realizada de maneira preventiva, a fim de garantir a manutenção da sua qualidade (BIONDI, 2010).

Sendo assim, o Cr pode levar a diversas alterações na qualidade do solo e das culturas sendoa determinação do Valor de Prevenção (VP) de grande importância para o gerenciamento de áreas contaminadas, sendo de caráter preventivo, utilizado para indicar alterações da qualidade do solo que possam prejudicar sua funcionalidade, e regular a introdução de substâncias químicas.

Dessa forma, torna-se importante a definição de valores orientadores para cada estado, em virtude de suas peculiaridades geomorfológicas, pedológicas e geológicas, em concordância com a Resolução $n^{\circ} 420$ do CONAMA. Este trabalho visa à investigação dos valores de prevenção para $\mathrm{Cr}$ em duas classes de solos representativas do Estado de Minas Gerais, como subsídio para a revisão de critérios regionais de prevenção

\section{MATERIAL E MÉTODOS}

O experimento foi conduzido em casa de vegetação, segundo as recomendações da Resolução CONAMA 420 de 2009, ISO 11.269-2. Foram selecionadas duas classes de solo do Estado de Minas Gerais consideradas representativas. Estas foram selecionadas usando como base o mapa de solos do Estado de Minas Gerais e os relatórios do Projeto Solos de Minas da FEAM, para os parâmetros classe de solo e mineralogia (FEAM, 2010).

Os solos selecionados para serem utilizados no experimento foram escolhidos por apresentarem os seguintes critérios: baixo teor de metais (abaixo do VRQ para todos); baixo teor de matéria orgânica (conteúdo de $\mathrm{C}$ orgânico não excede $15 \mathrm{~g} \mathrm{~kg}^{-1}$ ); mineralogia similar à mineralogia predominante no Estado de Minas Gerais; textura média; pH adequado (entre 5,5 e $6,6)$ de modo a minimizar os efeitos de adsorção de $\mathrm{Cr}$ no solo. Seguindo tais diretrizes, foram utilizados os horizontes superficiais dos seguintes solos: Latossolo Vermelho Amarelo distrófico A moderado textura média fase floresta tropical subperenifólia (LVAd) proveniente do Município de Itumirim-MG (SAD 69 23K 521.209 mE 7.646.209 mN a 874 m de altitude); e Cambissolo Háplico Tb distrófico típico A moderado textura argilosa (CXbd) (23K 501.439 mE 7.652.434 mN a $881 \mathrm{~m}$ de altitude) proveniente do Município de Lavras-MG.

As amostras de solos foram coletadas na camada de 0-0,20 m de profundidade, sob vegetação natural não antropizada, sem uso de fertilizantes e agroquímicos pelo menos nos últimos cinco anos. Essas amostras foram destorroadas, secas ao ar e passadas em peneira com malha de $5 \mathrm{~mm}$ de abertura. Posteriormente, foram retiradas subamostras dos diferentes solos, passadas em peneira de $2 \mathrm{~mm}$ de abertura e realizada a caracterização química e física.

A concentração do $\mathrm{Cr}$ foi determinada de acordo com o método USEPA 3051 A (USEPA, 1999), conforme requerido na Resolução Conama 420 de 2009. As amostras de solo foram suavemente trituradas com um almofariz de porcelana. Após a caracterização química dos solos foi realizada a correção, aplicando-se $\mathrm{CaCO} 3$ e $\mathrm{MgCO} 3$ na relação $\mathrm{Ca} / \mathrm{Mg} \mathrm{3:1}$, segundo as recomendações da 5 a Aproximação (RIBEIRO; GUIMARÃES, ALVAREZ, 1999). O solo ficou incubado por 20 dias. Posteriormente, foi realizada uma adubação básica, respeitando o nível crítico 
requerido para solos do Estado de Minas Gerais (5a aproximação) e os níveis mínimos requeridos para adubação em vasos por Malavolta (1980). Foram fornecidos aos solos, via solução nutritiva, os nutrientes $\mathrm{K} \mathrm{e} \mathrm{P}$, respectivamente nas seguintes quantidades $150 \mathrm{mg} \mathrm{kg}-1$ e $200 \mathrm{mg} \mathrm{kg}^{-1} \mathrm{via} \mathrm{KCl}$ e $\mathrm{NH} 4 \mathrm{H} 2 \mathrm{PO} 4$, respectivamente. A tabela 1 traz as características químicas dos solos usados no experimento após correção química.

Tabela 1: Características químicas da amostra de material dos solos utilizados, tratados com $\mathrm{CaCO}_{3}$ e $\mathrm{MgCO}_{3} \mathrm{e}$ adubados com KCl e $\mathrm{NH}_{4} \mathrm{H}_{2} \mathrm{PO}_{4}$. Departamento de Ciência do Solo da UFLA, 2013.

\begin{tabular}{|c|c|c|}
\hline Características* & LVAd & CXbd \\
\hline $\mathrm{pH}\left(\mathrm{H}_{2} \mathrm{O}\right)$ & 5,3 & 5,6 \\
\hline P mehlich $\left(\mathrm{mgdm}^{-3}\right)$ & 1,7 & 2,6 \\
\hline P-rem $\left(\mathrm{mgdm}^{-3}\right)$ & 19,6 & 5,3 \\
\hline $\mathrm{K}\left(\mathrm{mg} \mathrm{dm}^{-3}\right) \mathrm{Ca}$ & 38,0 & 64,0 \\
\hline$\left(\mathrm{cmol}_{\mathrm{c}} \mathrm{dm}^{-3}\right) \mathrm{Mg}$ & 0,5 & 4,5 \\
\hline$\left(\mathrm{cmol}_{\mathrm{c}} \mathrm{dm}^{-3}\right) \mathrm{Al}$ & 0,1 & 0,3 \\
\hline$\left(\mathrm{cmol}_{c} \mathrm{dm}^{-3}\right)$ & 0,6 & 0,2 \\
\hline $\mathrm{H}+\mathrm{Al}\left(\mathrm{cmol}_{C} \mathrm{dm}^{-3}\right)$ & 7,0 & 2,9 \\
\hline $\mathrm{SB}\left(\mathrm{cmol}_{\mathrm{c}} \mathrm{dm}^{-3}\right)$ & 0,7 & 5,0 \\
\hline C orgânico & 8,8 & 2,2 \\
\hline $\mathrm{V}(\%)$ & 9,0 & 34,0 \\
\hline $\mathrm{t}\left(\mathrm{cmol}_{\mathrm{c}} \mathrm{dm}^{-3}\right)$ & 1,3 & 5,2 \\
\hline $\mathrm{T}\left(\mathrm{cmol}_{\mathrm{c}} \mathrm{dm}^{-3}\right)$ & 7,7 & 7,9 \\
\hline m (\%) & 46,0 & 19,3 \\
\hline $\mathrm{S}\left(\mathrm{mg} \mathrm{dm} \mathrm{m}^{-3}\right)$ & 15,2 & 16,3 \\
\hline $\mathrm{Zn}\left(\mathrm{mg} \mathrm{dm} \mathrm{m}^{-3}\right)$ & 1,5 & 1,6 \\
\hline $\mathrm{B}\left(\mathrm{mg} \mathrm{dm} \mathrm{m}^{-3}\right)$ & 0,3 & 0,2 \\
\hline $\mathrm{Fe}\left(\mathrm{mg} \mathrm{dm^{-3 } )}\right.$ & 1190,0 & 102,0 \\
\hline $\mathrm{Mn}\left(\mathrm{mg} \mathrm{dm}^{-3}\right)$ & 11,6 & 18,6 \\
\hline $\mathrm{Cu}\left(\mathrm{mg} \mathrm{dm} \mathrm{m}^{-3}\right)$ & 2,0 & 2,1 \\
\hline
\end{tabular}

A granulometria dos solos foi determinada pelo método da pipeta (DAY, 1965) empregando-se $\mathrm{NaOH} \mathrm{0,1} \mathrm{mol} \mathrm{L-1} \mathrm{como} \mathrm{dispersante} \mathrm{químico} \mathrm{e} \mathrm{agitação} \mathrm{rápida,} \mathrm{sendo} \mathrm{a} \mathrm{fração} \mathrm{areia}$ (2- 0,03 mm) separada por meio de peneira, os resultados encontram-se na Tabela 2.

Tabela 2: Valores de textura e classificação textural (CT) de CXbd e LVAd, retiradas na camada de 0 a $20 \mathrm{~cm}$ de profundidade. Departamento de Ciência do Solo da UFLA, 2013

\begin{tabular}{c|c|c}
\hline & LVAd & CXbd \\
\hline Argila $\left(\mathrm{g} \mathrm{kg}^{-1}\right)$ & 260 & 460 \\
Areia $\left(\mathrm{g} \mathrm{kg}^{-1}\right)$ & 700 & 350 \\
Silte $\left(\mathrm{g} \mathrm{kg}^{-1}\right)$ & 40 & 190 \\
CT & Textura Média & Argila Arenosa \\
\hline
\end{tabular}

A concentração do $\mathrm{Cr}$ foi determinada de acordo com o método USEPA 3051 A (USEPA, 1999), conforme requerido na Resolução Conama 420 de 2009. As amostras de solo foram suavemente trituradas com um almofariz de porcelana. Neste método, quatro repetições de $0,5 \mathrm{~g}$ de solo foram digeridas em $5 \mathrm{ml}$ de HNO3 concentrado e $5 \mathrm{~mL}$ de água tridestilada, em frascos de 
Teflon hermerticamente fechados, aquecidos em forno de micro-ondas por 10 minutos. Para a caracterização do solo, foi realizada leitura do elemento $\mathrm{Cr}$ determinado em espectrofotômetro de absorção atômica em chama de ar-acetileno. O teor médio do Cr para o LVAd é de $16,96 \mathrm{mg} \mathrm{kg}^{-1}$ de solo seco e para o CXbd é de 23,10 de solo seco, $\mathrm{N}$ valores estes certificados do padrão Montana I Soil 2710a.

O ensaio fitotoxicológico foi realizando conforme a norma ISO 11.269-2:2012. Duas espécies de plantas superiores foram selecionadas a partir da lista de espécies sugeridas pela ISO 11269-2: a espécie monocotiledônea milho (Zea mays) e dicotiledônea Feijão (Phaseolus aureus cv Carioquinha). As sementes de ambas foram adquiridas a partir de fontes comerciais. Estas foram escolhidas, dentre outros fatores, por serem plantas de ciclo anual e de rápido crescimento, além de amplamente cultivadas em todo o Brasil. Os tratamentos foram constituídos de oito doses de Cr no solo $\left(0,5,10,20,45,90,200,400 \mathrm{mg} \mathrm{kg}^{-1}\right)$. Os valores estabelecidos são aproximadamente múltiplos de 1,8 conforme sugestão da ISO 11269-2. Após os solos devidamente corrigidos, foram adicionadas soluções aquosas de dicromato de K, variando de acordo com o tratamento para duas classes de solo LVAd e CXbd.

Após 24 horas da incorporação do dicromato de K, dez sementes foram semeadas. A dose de $\mathrm{K}$ foi parcelada no primeiro e no décimo dia do experimento para evitar uma possível salinização. A irrigação de cada vaso foi feita por capilaridade com utilização de água destilada. Para isso, o vaso contendo o solo foi furado no fundo e por ele passado uma corda de seda trançada que foi imersa em outro recipiente, montado abaixo do vaso, contendo água. Assim, a água subia por capilaridade pela corda até o recipiente contendo solo.

Com este método, foi possível reduzir o período de rega e manter o fornecimento de água homogêneo para as plantas. O teor de umidade de cada vaso foi verificado a cada três dias, pesando recipientes (ALEXANDRINO, 2014). Ao final de 21 dias após a emergência de $70 \%$ do grupo controle, foram avaliadas as seguintes variáveis: altura da parte aérea, comprimento de raiz, massa seca da parte aérea e massa seca de raiz. A altura das plantas foi medida por paquímetro, a massa seca da parte aérea e da raiz foi obtida após serem secas em estufa a $75^{\circ} \mathrm{C}$ por $16 \mathrm{~h}$, obtendo-se peso constante.

Os dados obtidos nos ensaios com as plantas foram analisados utilizando o programa Statistica 7.0. As variáveis massa seca e altura de plantas foram analisadas estaticamente por ANAVA, seguindo-se o teste de regressão. Os valores de ECx foram calculados utilizando os modelos log exponencial, modelo Gompertz, modelo Hormesis e modelo Logistic, sendo utilizado o modelo que apresentou o melhor valor de R2 e significância em 95\% (VLAARDIGEN, 2004). Estes modelos estatísticos foram utilizados apenas para o conjunto de dados caracterizado por tendências decrescentes dos valores de ECx. ECx é definida como a concentração que reduz a taxa de reprodução em uma determinada porcentagem em relação ao controle. Os modelos logístico, exponencial, hormesis e gompertz foram os que mais se adequaram e também os mais recomendados para explicar o comportamento dos dados de EC50 e EC5, as equações desses modelos são as seguintes:

EC50:Variável Resposta $=\mathrm{t} /(1+(\mathrm{conc} / \mathrm{X}) \mathrm{b}$

EC5: Variável Resposta $=t /(1+(0,2 / 0,8) \times($ conc/X) b $)$

- Modelo Logistic: 
EC50: Variável Resposta $=a \times \exp (\log ((a-a \times 0,5-b \times 0,5) / a) \times($ conc/X)) +b

EC5: Variável Resposta $=a \times \exp (\log ((a-a \times 0,2-b \times 0,8) / a) \times(\operatorname{conc} / X))+b$

- Exponencial:

IC50: Variável Resposta $=(\mathrm{t} \times(1+\mathrm{h} \times$ logconc $)) /(1+((0,5 \times($ logconc $) / 0,5) \times($ logconc $/ \mathrm{X}) \mathrm{b})$

IC5: Variável Resposta $=(\mathrm{t} \times(1+\mathrm{h} \times$ logconc $)) /(1+((0,25 \times($ logconc $) / 0,75) \times($ logconc $/ \mathrm{X}) \mathrm{b})$

- Modelo Hormesis:

EC50: Variável Resposta $=\mathrm{g} \times \exp ((\log (0,5)) \times($ conc/X) b )

EC5: Variável Resposta $=g \times \exp ((\log (0,8)) \times(\operatorname{conc} / X) b)$

- Modelo Gompertz:

Onde:

b = Parâmetro estimado entre 1 e 4

$\mathrm{X}=\mathrm{ICP}$ para um conjunto de dados

$\log c o n c=$ concentração de exposição, transformada em log

a, g ou $\mathrm{t}=$ interseção y (resposta do controle)

Para obter o valor de prevenção para solos deve-se encontrar valores de EC50 e EC5 para cada concentração teste, utilizando plantas e organismos do solo recomendadas pelas normas internacionais e que ao mesmo tempo sejam representativas para as regiões de estudo. Após os ECx serem determinados, o valor de HC5 e HC50 pode ser calculado para cada solo avaliado. Como os valores de $\mathrm{HC} 5$ são mais protetores que os de $\mathrm{HC50}$, os primeiros se tornam normalmente mais recomendáveis para se estabelecer o valor de VP.Após os HC5 estabelecidos, estes serão somados ao VRQ (VLAARDINGEN, 2004).

\section{RESULTADO E DISCUSSÃO}

O gráfico $1 \mathrm{~A}$ e $1 \mathrm{~B}$ trazem as diferentes concentrações de $\mathrm{Cr}$ na parte aérea e raiz para a cultura do milho e feijão, cultivados em LVAd, resultado da análise de regressão.

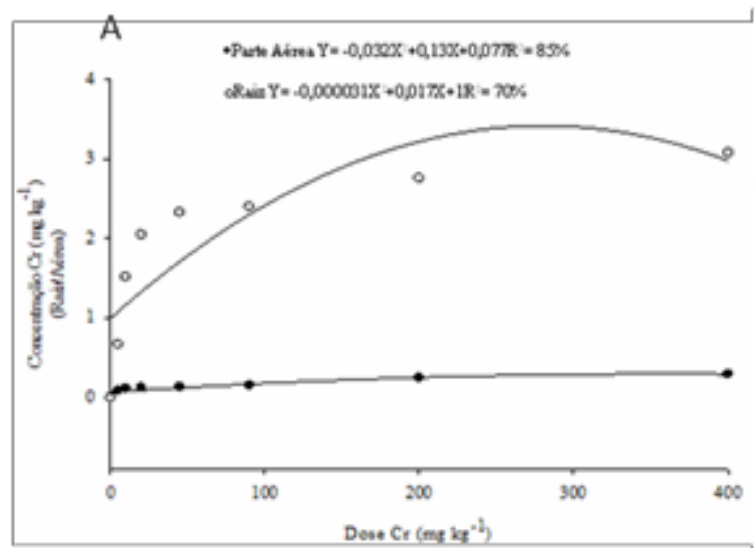

a)

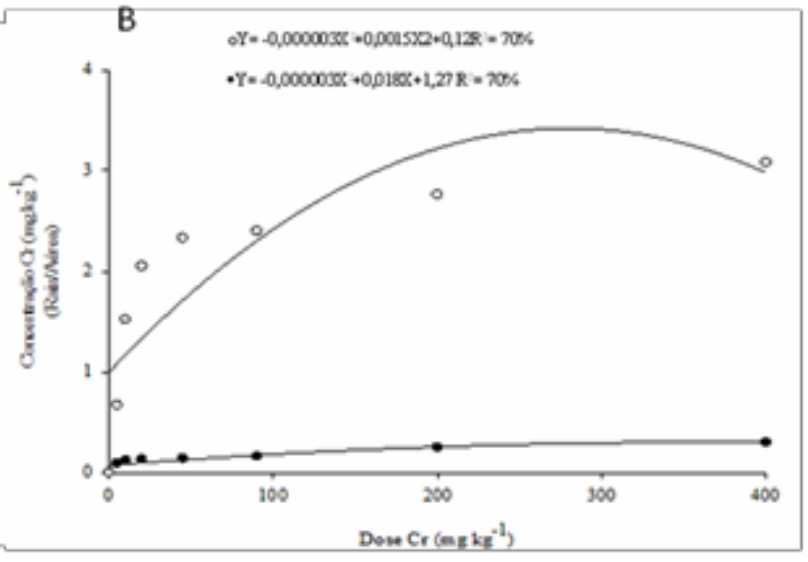

b)

Figura 1: Diferentes concentrações de Cr na parte aérea e raiz - a) cultura do milho cultivado em LVAd; b) cultura do feijão cultivado em LVAd. 
Nota-se que quanto maior a dose de $\mathrm{Cr}$ no solo maior a concentração deste na parte aérea e raiz do milho e feijão. Todas as doses apresentaram $\mathrm{Cr}$ em suas massas secas de raiz e parte aérea, porém a massa seca de raiz apresentou valores superiores. A maioria das pesquisas com $\mathrm{Cr}$ mostram que este é acumulado principalmente nas raízes, sendo uma pequena parte translocada para os órgãos aéreos. Isso se deve à imobilização do $\mathrm{Cr}$ nos vacúolos das células radiculares, reduzindo seus efeitos tóxicos, o que pode ser uma resposta natural da planta a toxidez (SHANKER et al., 2005).

No gráfico 2A e 2B, são apresentados as diferentes concentrações de $\mathrm{Cr}$ na parte aérea e raiz para a cultura do milho e feijão, cultivados em CXbd, resultado da análise de regressão quadrática, com significância de 1\%, para todas as doses e para as duas culturas apresentadas.

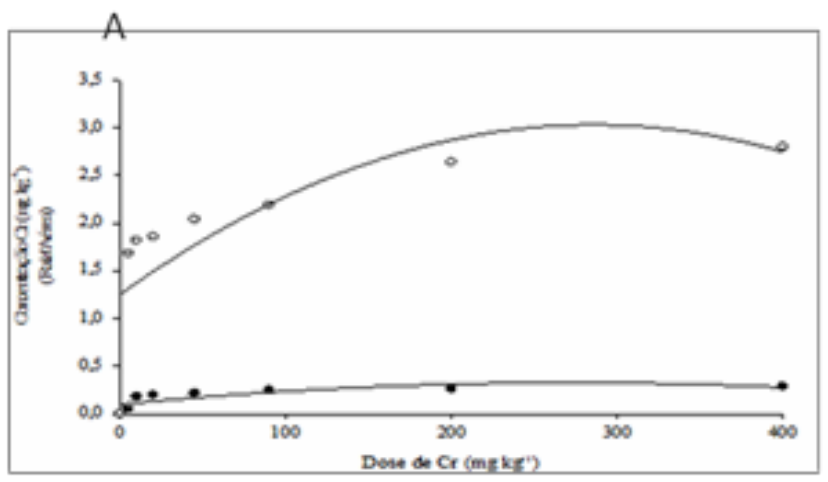

a)

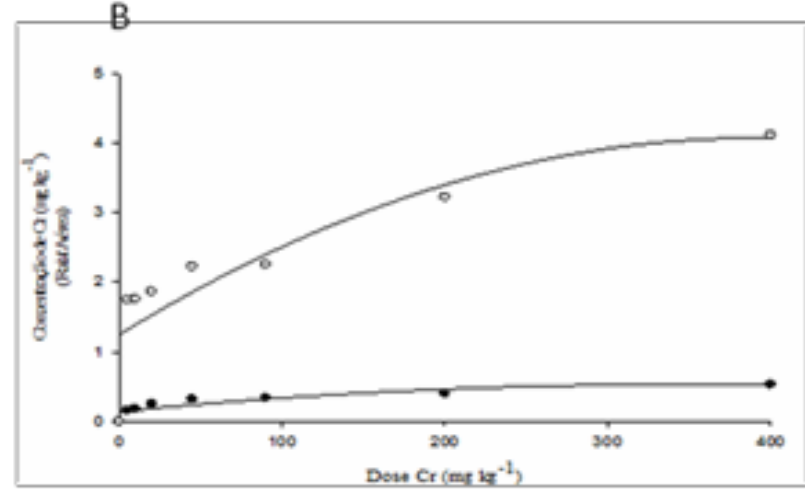

b)

Figura 2: Diferentes concentrações de Cr na parte aérea e raiz - a) cultura do milho cultivado em CXbd; b) cultura do feijão cultivado em CXbd.

Os teores de $\mathrm{Cr}$ nas massas secas de raiz e parte aérea foram maiores conforme as doses aumentaram, e as massas secas de raízes foram superiores quando comparadas com suas partes aéreas, independente das culturas. Srivastava et al. (1999), estudando a distribuição de $\mathrm{Cr}$ nos tecidos vegetais do tomateiro (raiz, parte aérea e frutos), observaram que 80 a $85 \%$ do mesmo é retido nas raízes e somente uma pequena fração é translocada para a parte aérea.

Huffman e Allaway (1990) também observaram que mais de $90 \%$ do $\mathrm{Cr}$ absorvido em plantas de cevada e de trigo fica retido nas raízes, explicando desta forma as massas secas de raízes apresentarem valores superiores as massas secas de parte aérea. Os estudos da interação entre plantas e $\mathrm{Cr}$ na rizosfera são relativamente limitados (Cary e Kubota, 1990; Chang et al., 1992; Bishnoi et al., 1993; Corradi et al., 1993; Han et al., 2004; Weis e Weis, 2004).

No entanto, as condições ambientais da rizosfera podem afetar o potencial do impacto das raízes na mobilidade do $\mathrm{Cr}$ (Terry, 2003). A presença de exsudatos radiculares pode favorecer condições de redução do solo, imobilizando o cromo (Srivastava et al., 1999). Por outro lado, raízes podem liberar agentes complexantes, facilitando a absorção e a translocação do cromo para a parte aérea de plantas ou o aumento da mobilidade do $\mathrm{Cr}$ no solo.

Para os dois solos e ambas as culturas, os teores de $\mathrm{Cr}$ encontrados em todos os tratamentos ficaram acima da faixa suficiente ou normal $\left(0,03\right.$ a $\left.14 \mathrm{mg} \mathrm{kg}^{-1}\right)$ para culturas agronômicas em tecido foliar e bem abaixo da faixa considerada tóxica (5 a $30 \mathrm{mg} \mathrm{kg}^{-1}$ ) para a maioria das plantas cultivadas (KABATA-PENDIAS; PENDIAS, 2001). 
Como apresentado na tabela 2, os solos selecionados apresentam concentração de $\mathrm{Cr}$ com valor abaixo dos limites legais, ou seja, do VRQ da Deliberação Normativa 02 de 2010 do Estado de Minas Gerais, sendo encontrado para LVAd 2,9 mg kg-1 e no CXbd 4,7 mg kg${ }^{-1}$. Na tabela 3, são apresentadas as médias dos valores de EC50 e os valores do coeficiente de determinação dos modelos matemáticos. Pode-se observar que os parâmetros avaliados tiveram comportamento estatístico não linear.

Tabela 3: Valores de EC50 para LVAd e CXbd. Departamento de Ciência do Solo da UFLA, 2013

\begin{tabular}{c|c|c|c|c}
\hline Variável & Espécie & $\mathrm{EC50} \mathrm{mg} \mathrm{kg}^{-1}$ & Modelo Estatístico & $\mathrm{R}^{2}(\%)$ \\
\hline LVAd & & & & \\
\hline Alt PA & Feijão & 89,98 & Exponencial & 89 \\
CR & Feijão & 124,4 & & 97 \\
\hline CXbd & & & & 87 \\
& & $3.351,59$ & Hormesis & 98 \\
MSPA & Feijão & 6258,46 & Hormesis Hormesis & 98 \\
Alt PA Alt PA CR & Feijão Milho & 3488,04 & Logistic Hormesis & 70 \\
MSPA MSR & Milho Milho & 26415,78 & Hormesis & 97 \\
& Milho & 1513,1 & & 94 \\
\hline
\end{tabular}

Para obterem-se os valores de EC50, é necessário usar como dados as variáveis das culturas avaliadas (milho e feijão), tais como altura da parte aérea (Alt PA), comprimento de raiz (CR), massa seca de parte aérea (MSPA), massa seca de raiz (MSR). Quando estatisticamente analisadas, é importante que as diferenças entre tratamentos sejam significativas. Contudo, para o LVAd, na cultura do milho e na cultura do feijão (apenas nas variáveis MSR e MSPA), tais diferenças não apresentaram significância. Portanto, não foi possível determinar um VP para o LVAd. Para o CXbd, na cultura do feijão,asvariáveis também não apresentaram significância estatística, sendo por este motivo não apresentadas na tabela 3. Valores de EC50 menores são considerados ambientes mais sensíveis. Ao utilizar EC50 para umavariável específica, estamos estimando a concentração na qual a variável está sendo reduzida em 50 \% em relação ao grupo controle. Para o CXbd, na cultura do feijão, valores muito maiores de EC50 foram encontrados para massa seca da parte aérea e altura da parte aérea sendo estes $3.352 \mathrm{mg} \mathrm{kg}^{-1}$ e $6.258 \mathrm{mg} \mathrm{kg}^{-1}$, respectivamente. Isso quer dizer que mesmo nestas altíssimas concentrações as plantas apresentaram reduções de 50 \% em seu desenvolvimento.

Numa avaliação de risco fitotoxicológica, um ecossistema é considerado protegido quando as espécies em questão possuem $95 \%$ das variáveis usadas para calcular seus respectivos ECX protegidas. Para tanto, de posse dos valores de EC50, foram plotados gráficos de distribuição das variáveis estudadas de acordo com a sensibilidade (SSD) (Gráfico 3). A partir de então, foram obtidos os valores da concentração limite para $5 \%$ das variáveis testadas (HC5), que é a restrição necessária para a proteção do ecossistema em um solo que está em monitoramento e sob alerta. 


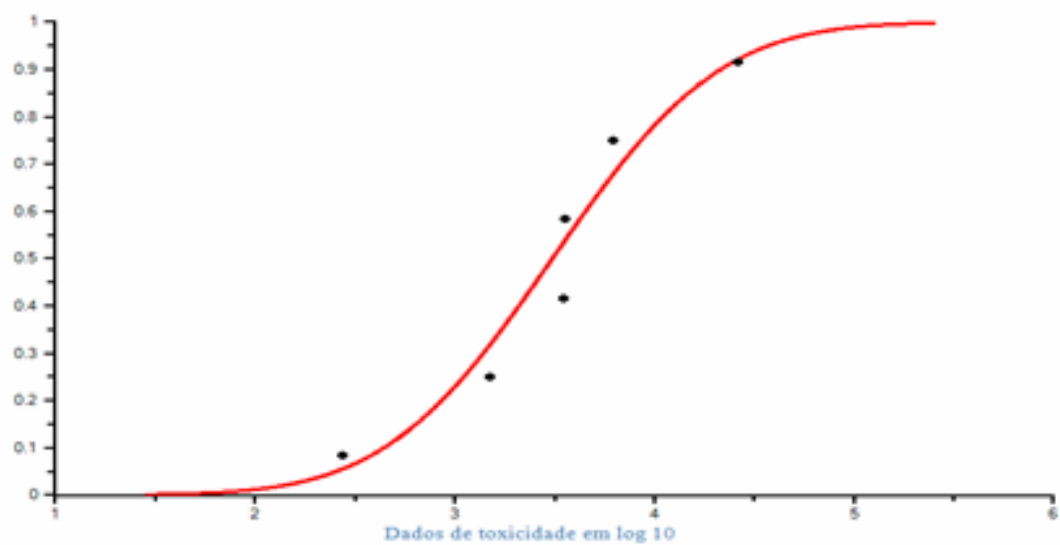

Figura 3: Distribuição da sensibilidade das espécies para EC50 (concentração efetiva que causa danos a $50 \%$ das espécies) para CXbd.

O cálculo do valor de HC5 permite derivar um valor de alerta, enquanto que o cálculo do valor de HC50, menos restritivo, permite derivar, por exemplo, um valor de investigação agrícola. Essas curvas de sensibilidade permitem utilizar valores mais ou menos restritivos para uma avaliação de risco ecotoxicológico ou no gerenciamento de áreas contaminadas. É possível estabelecer HCs para diferentes níveis (exemplo 10\%, 20\%, 50\%, 70\% etc.) dependendo do nível que se quer proteger e qual cenário abranger (agrícola, residencial ou industrial). Neste estudo, os valores de $\mathrm{HC} 5$ e HC50 foram baseados nos valores de EC50 obtidos nos ensaios de fitotoxicidade, cujos valores são apresentados na tabela 3 . Visando proteger as espécies mais sensíveis, o VP originado do HC5 é mais recomendado por ser mais preventivo que o valor HC50. Logo é mais seguro trabalhar com HC5 para derivar VP.

O valor de HC5 para CXbd foi de $217 \mathrm{mg} \mathrm{kg}^{-1}$. Isso quer dizer que, até a concentração de $217 \mathrm{mg} \mathrm{kg}^{-1}, 95 \%$ das variáveis estudadas para a cultura do milho estarão protegidas (i.e.,terão reduções em seu desenvolvimento inferiores a $50 \%$ ); Ao passo que 5\% das variáveis haverá provavelmente redução no desenvolvimento superior a $50 \%$.

Somando-se o HC50 obtido neste trabalho com o VRQ, encontrou-se neste trabalho um VP de $292 \mathrm{mg} \mathrm{kg}^{-1}$. O valor atual preconizado pela legislação é de $75 \mathrm{mg} \mathrm{kg}^{-1}$. Para obter-seum VP abrangente e efetivo é obviamente necessário avaliar não só avaliarvariáveis oriundas de plantas superiores, como feito neste trabalho, mas também avaliar o efeito da substância tóxica em outros organismos do solo (minhocas, p.e.), o que não foi feito neste trabalho. Não obstante, o VP encontrado neste estudo serve como uma referência para estudos com outros organismos de solo submetidos à toxidez de $\mathrm{Cr}$.

\section{CONCLUSÃO}

Pode-se afirmar que quanto maior as doses de $\mathrm{Cr}$ adicionadas nos solos maiores os teores de $\mathrm{Cr}$ presentes na planta. Apesar das culturas absorverem $\mathrm{Cr}$ em suas estruturas, grande parte deste fica ainda retido solo. O valor de VP estimado encontrado neste trabalho foi de $292 \mathrm{mg} \mathrm{kg}^{-1}$. Como o valor atualmente preconizado pela legislação é de $75 \mathrm{mg} \mathrm{kg}^{-1}$, este trabalho aponta para a necessidade de revisão do valor estabelecido pela Deliberação Normativa Conjunta COPAM/CERHMG no 02, de 08-09-2010. 


\section{REFERÊNCIAS BIBLIOGRÁFICAS}

1. ALEXANDRINO, R. C. S. Determinação do valor de prevenção para solos contaminados por chumbo no estado de Minas Gerais. 2014. 146 p. Dissertação (Mestrado em Ciências do Solo) - Universidade Federal de Lavras, Lavras, 2014.

2. ALLOWAY, B. J. Heavy metals in soils. 2. ed. Londres: Springer, 1990. 363 p.

3. BIONDI, C. M. Teores naturais de metais pesados nos solos de referência do Estado de Pernambuco. Doctor, 1-46, 2010.

4. BISHNOI, N. R. et al. Effect of chromium on seed germination, seedling growth and yield of peas. Agriculture, Ecosystems and Environment, Amsterdam, v. 47, n. 1, p. 47-57, Oct. 1993.

5. CARY, E. E.; KUBOTA, J. Chromium concentration in plants: effects of soil chromium concentration and tissue contamination by soil. Journal of Agriculture and Food Chemistry, Easton, v. 38, n. 1, p. 108-114, Jan. 1990.

6. CHANG, A. C.; WARNEKE, J. W.; PAGE, A. L. Accumulation of heavy metals in sewage sludgetreated soils. Journal of Environmental Quality, Madison, v. 13, n. 1, p. 87-91, Jan. 1992.

7. CONSELHO NACIONAL DO MEIO AMBIENTE. Resolução no 420, de 28 de dezembro de 2009. Dispõe sobre critérios e valores orientadores de qualidade do solo quanto à presença de substâncias químicas e estabelece diretrizes para o gerenciamento ambiental de áreas contaminadas por essas substâncias em decorrência de atividades antrópicas. Brasília, 2009. Disponível em: <http://www.mma.gov.br/port/conama/res/res09/res42009.pdf>. Acesso em: 10 dez. 2015.

8. CORRADI, M. G.; BIANCHI, A.; ALBASINI, A. Chromium toxicity in Saliviasclarea: I effects of hexavalent chromium on seed germination and seedling development. Environmental and Experimental Botany, Paris, v. 33, p. 405- 413, 1993.

9. DAY, P. R. Particle fractionation and particle-size analysis. In: BLACK, C. A. (Ed.). Methods of soil analysis: physical and mineralogical properties including statistics of measurement and sampling: part 1. Madison: American Society of Agronomy, 1965. p. 545-567.

10. DETTMER, A. et al. Obtaining sodium chromate from ash produced bey thermal treatment of leather waster. Chemical Engineering Jornal, Lausanme. V.160, Ap 2010.

11. FUNDAÇÃO ESTADUAL DE MEIO AMBIENTE - FEAM. Mapa de solos do Estado de Minas Gerais: legenda expandida / Universidade Federal de Viçosa; Fundação Centro Tecnológico de Minas Gerais; Universidade Federal de Lavras; Fundação Estadual do Meio Ambiente. Belo Horizonte, 2010. 49 p.

12. FUNDAÇÃO ESTADUAL DE MEIO AMBIENTE - FEAM. Inventário de Áreas Suspeita de Contaminação e contaminadas do Estado de Minas Gerais - 2010. Disponível em: > www.feam.br < Acesso em 14 julho. 2014.

13. FUNDAÇÃO ESTADUAL DE MEIO AMBIENTE - FEAM. Mapa de solos do Estado de Minas Gerais: legenda expandida / Universidade Federal de Viçosa; Fundação Centro Tecnológico de Minas Gerais; Universidade Federal de Lavras; Fundação Estadual do Meio Ambiente. Belo Horizonte, $2010.49 \mathrm{p}$.

14. HAN, F. X.; SRIDHAR, B. B.; MONTS, D. L. Phytoavailability and toxicity of trivalent and hexavalent chromium to Brassica juncea. New Phytologist, Baltimore, v. 162, n. 2, p. 489-499, May 2004.

15. HUFFMAN JÚNIOR, E. W. D.; ALLAWAY, H. W. Chromium in plants: distribution in tissues, organelles, and extracts and availability of bean leaf $\mathrm{Cr}$ to animals. Journal Agricultural and Food Chemistry, Easton, v. 21, n. 6, p. 982-986, Nov./Dec. 1973. 
16. INTERNATIONAL ORGANIZATION FOR STANDARDIZATION.ISO 11268-1.Soil quality - Effects of polutants on earthworms (Eiseniafetida) - Part 1: determination of acute toxicity using artificial soil substrate.Geneva, 1993.

17. INTERNATIONAL ORGANIZATION FOR STANDARDIZATION.ISO 11268-2.Soil quality - Effects of polutants on earthworms (Eiseniafetida) - Part 2: determination of effects on reproduction. Geneva, 1998

18. INTERNATIONAL ORGANIZATION FOR STANDARDIZATION.ISO 11269-2: soil quality: determination of the effects of pollutants on soil flora: part 2, effects of contaminated soil on the emergence and early growth of higher plants. Geneva, 2012. 19 p.

19. KABATA-PENDIAS, A. Trace Elements in Soil and Plants. 4rded. New York, Boca Raton, 2011. $467 \mathrm{p}$.

20. KABATA-PENDIAS, A.; PENDIAS, H. Trace elements in soils and plants.Boca Raton: CRC Press, 2001. $432 \mathrm{p}$.

21. MALAVOLTA, E. Elementos de nutrição mineral de plantas. São Paulo: Agronômica Ceres, 1980. $215 \mathrm{p}$.

22. MALAVOLTA, E. Manual de nutrição mineral de plantas. São Paulo: Agronômica Ceres, 2006. $631 \mathrm{p}$.

23. MARTINES -LLADO, X., VILA, M., MARTI, V., ROVIRA, M., DOMENECH, J.A., DE PABLJO, J. Trace element distribution in topsoils in Catalonia: Background and reference values and relationship with regional geology. Environmental Engineering Science 25: 863-878, 2008.

24. MINAS GERAIS, CONSELHO ESTADUAL DE POLÍTICA AMBIENTAL - COPAM; CONSELHO ESTADUAL DE RECURSOS HÍDRICOS - CERH/MG. Deliberação Normativa Conjunta COPAM/CERH-MG n 02, de 08 de setembro de 2010. Institui o Programa Estadual de Gestão de Áreas Contaminadas, que estabelece as diretrizes e procedimentos para a proteção da qualidade do solo e gerenciamento ambiental de áreas contaminadas por substâncias químicas.

25. PELOZATO, M. Valores de referência de cádmio, cobre, manganês e zinco para solos de Santa Catarina. 2008. 69 p. Dissertação (Mestrado em Manejo do Solo). Universidade do Estado de Santa Catarina, Lages, 2008.

26. RIBEIRO, A. C.; GUIMARÃES, P. T. G.; ALVAREZ V., V. H. (Ed.). Recomendação para o uso de corretivos e fertilizantes em Minas Gerais: 5. Aproximação. Viçosa: Comissão de Fertilidade do Solo do Estado de Minas Gerais, 1999. 359p.

27. SHANKER, A. K. et al. Chromium toxicity in plants.Environment International, New York, $\mathrm{n}$. 32.n.5, p.739-753, July 2005.

28. SRIVASTAVA, S.; PRAKASH, S.; SRIVASTAVA, M. M. Chromium mobilization and plant availability: the impact of organic complexing ligands. Plant and Soil, The Hague, v. 212, n. 2, p. 203-208, May 1999.

29. UNITED STATES ENVIRONMENTAL PROTECTION AGENCY. 1998 update of ambient water quality criterion for ammonia. Washington: Office of Water, 1999. $153 \mathrm{p}$.

30. VLAARDINGEN, P. VAN et al. ETX 2.0: a program to calculate hazardous concentrations and fraction affected, based on normally distributed toxicity data: RIVM Report $\mathrm{n}$ ㅇ 601501028/2004. Bilthoven: RIVM, 2004. 68 p.

31. WEIS, J. S.; WEIS, P. Metal uptake, transport, and release by wetland plants: implications for phytoremediation and restoration. Environment International, Oxford, v. 30, n. 5, p. 685-700, July 2004.

32. TERRY, N. Chromium in the environment: factors affecting biological remediation. Plant and Soil, The Hague, v. 249, p. 139-156, 2003. 